\title{
Telomere shortening and telomere position effect in mild ring 17 syndrome
}

\author{
Cecilia Surace ${ }^{1 *}$, Francesco Berardinellii ${ }^{2+}$, Andrea Masotti ${ }^{3}$, Maria Cristina Roberti ${ }^{1}$, Letizia Da Sacco ${ }^{3}$, \\ Gemma D'Elia', Pietro Sirleto ${ }^{1}$, Maria Cristina Digilio ${ }^{4}$, Raffaella Cusmai ${ }^{5}$, Simona Grotta ${ }^{1}$, Stefano Petrocchi ${ }^{1}$, \\ May El Hachem ${ }^{6}$, Elisa Pisaneschi ${ }^{1}$, Laura Ciocca', Serena Russo ${ }^{1}$, Francesca Romana Lepri ${ }^{1}$, Antonella Sgura ${ }^{2}$ \\ and Adriano Angioni ${ }^{1}$
}

\begin{abstract}
Background: Ring chromosome 17 syndrome is a rare disease that arises from the breakage and reunion of the short and long arms of chromosome 17. Usually this abnormality results in deletion of genetic material, which explains the clinical features of the syndrome. Moreover, similar phenotypic features have been observed in cases with complete or partial loss of the telomeric repeats and conservation of the euchromatic regions. We studied two different cases of ring 17 syndrome, firstly, to clarify, by analyzing gene expression analysis using real-time qPCR, the role of the telomere absence in relationship with the clinical symptoms, and secondly, to look for a new model of the mechanism of ring chromosome transmission in a rare case of familial mosaicism, through cytomolecular and quantitative fluorescence in-situ hybridization (Q-FISH) investigations.
\end{abstract}

Results: The results for the first case showed that the expression levels of genes selected, which were located close to the $\mathrm{p}$ and $\mathrm{q}$ ends of chromosome 17, were significantly downregulated in comparison with controls. Moreover, for the second case, we demonstrated that the telomeres were conserved, but were significantly shorter than those of age-matched controls; data from segregation analysis showed that the ring chromosome was transmitted only to the affected subjects of the family.

Conclusions: Subtelomeric gene regulation is responsible for the phenotypic aspects of ring 17 syndrome; telomere shortening influences the phenotypic spectrum of this disease and strongly contributes to the familial transmission of the mosaic ring. Together, these results provide new insights into the genotype-phenotype relationships in mild ring 17 syndrome.

Keywords: Genetic syndrome, Telomere position effect, Ring 17 chromosome, Telomere shortening

\section{Background}

Ring chromosomes are unusual circularized chromosomes, the vast majority of which arise sporadically [1]. Two types of ring chromosome can be related to different clinical presentations: the non-supernumerary ring, which replaces one of the normal homologs, with the karyotype 46,(r) and the supernumerary ring, which is often very small, with the karyotype $47,+(r)$. The non-supernumerary ring chromosomes are usually associated with abnormal phenotypes because of loss of genomic material at one or both ends. In

\footnotetext{
* Correspondence: cecilia.surace@opbg.net

${ }^{\dagger}$ Equal contributors

'Cytogenetics and Molecular Genetics Unit, 'Bambino Gesù' Children's

Hospital, IRCCS, Piazza S. Onofrio 4, 00165 Rome, Italy

Full list of author information is available at the end of the article
}

some cases, no deletion has been detected and the phenotypic features have been attributed to mitotic ring instability [2].

The classic mode of ring chromosome formation consists of two terminal breaks in both chromosome arms, followed by a reconnection of the broken ends, leading to a loss of genetic material. Alternatively, they can be formed by fusion of subtelomeric sequences or telomere-telomere fusion with no deletion, resulting in complete ring chromosomes.

Telomeres, the nucleoprotein complexes that cap eukaryotic chromosomes, play two major roles in cell physiology. First, they are critical factors in determining the lifespan of mammalian cells. Second, they are essential for genome integrity maintenance. In addition to their role in 
protecting the ends of chromosomes, telomeres can influence the expression of nearby genes, an event called the telomere position effect. Previous reports demonstrated that the telomere position effect is an epigenetic process that involves changes in chromatin conformation and depends on both the distance from the telomere and the telomere's length [3]. The telomere position effect also occurs in mammalian cells, and may play a role in human genetic disease, as a result of the repositioning of active genes near telomeres or subtelomeric sequences following chromosome rearrangements [4-8], such as those seen in individuals with ring chromosomes $[9,10]$.

Rings of chromosome 17 are relatively rare and only 17 cases have been reported in the literature to date. Among these 17 cases, 11 showed no deletions of the Miller-Dieker critical region with mild phenotypic features [11] consisting of growth delay, intellectual disability, seizures, café au lait skin spots and minor facial dysmorphism.

The ring chromosomes 17 of the majority of these patients were described at the banding level, and only two cases pointed at the molecular level [11-13]. Cytomolecular investigation of the telomeric and subtelomeric regions of ring 17 is essential for the disclosure of candidate genes or gene expression regulating regions, which are potentially responsible for the phenotypic characteristics.

We report here the results of new investigations of a previously described patient with mild ring 17 syndrome and studies of an unusual case of ring chromosome 17 syndrome with a mosaic familial transmission.

\section{Results \\ Case 1 \\ Clinical presentation}

The clinical features of patient 1 have been previously reported [11]. We evaluated the patient at four years of age. Clinical examination showed prominent eyes, epicanthal folds, anteverted nares, thick lips, small teeth, prognathism, generalized café au lait skin spots, and hypochromic cutaneous lesions. Developmental milestones were moderately delayed and language was absent. The patient developed epilepsy when he was two years old: this was characterized by tonic seizures that were difficult to control by medical treatment. The patient's ductus arteriosus was closed by cardiac catheterization at five years of age. Cytogenetic investigations from peripheral blood cells revealed the following karyotype: 46, $\mathrm{XY}, \mathrm{r}(17)(\mathrm{p} 13 \mathrm{q} 25)[27] / 45, \mathrm{XY},-17[3]$. Chromosome analysis of fibroblasts obtained by dyschromic skin spot biopsy showed the sole ring 17 pattern without the monosomic cell line. Fluorescence in-situ hybridization (FISH) investigations revealed the loss of $r(17)$ telomeres and conservation of the whole adjacent, euchromatic, subtelomeric regions.

\section{Gene expression analysis}

The expression levels of 24 selected genes within a $1.5 \mathrm{Mb}$ region starting from each telomere and one housekeeping gene $(G A P D H)$ of the ring 17 patient were measured in each sample by real-time qPCR and compared with controls. Two other genes (NF1 and LIS1) that are located outside the $1.5 \mathrm{Mb}$ region (17q11.2 and $17 \mathrm{p} 13.3$, respectively), were also considered. Of a total of 26 genes analyzed, 14 genes were statistically significant $(P<0.05)$, and 12 of these were located within the $1.5 \mathrm{Mb}$ region (Table 1 ). Of these latter genes, seven were found to be deregulated by a fold change (FC) greater than 1.5: RPH3AL, FAM57A, GEMIN4, $N X N$ in the sub-telomere region of $17 \mathrm{p}$ and $A R H G D I A$, $N A R F$, and FN3K located in the $17 \mathrm{q}$ arm. The FOXK2 gene displayed a downregulation that was below the FC threshold of $1.5(\mathrm{FC}=-1.44)$. The modulation of YWHAE, MYOC1, SIRT7, and RNMTL1 is negligible, suggesting that these genes are not sensitive to the telomere influence. Interestingly, the NF1 gene displayed a significant upregulation of 2.56, whereas LIS1 is downregulated -1.76 fold. Taken together, these results emphasize the downregulation of the majority of genes included within the investigated sub-telomere regions.

\section{Case 2 \\ Clinical presentation}

The patient, a girl, was the first child of unrelated parents. At birth, the mother was 27 years old and the father was 29. The baby was born at term by vaginal delivery after an uneventful pregnancy. The baby's birth weight was $3100 \mathrm{~g}$, she was $48.5 \mathrm{~cm}$ long, and the circumference of her head was $33.5 \mathrm{~cm}$. The patient was first evaluated at 2.4 years of age. Her weight was $12 \mathrm{~kg}$ (25th centile), she was $79 \mathrm{~cm}(<3 \mathrm{rd}$ centile) high, and her head circumference was $45 \mathrm{~cm}$ ( $<3 \mathrm{rd}$ centile). Clinical examination showed sparse café au lait spots on her trunk and legs, as in her mother. No facial anomalies were noted. Developmental milestones were mildly delayed (sitting at nine months, walking alone at 18 months). At six years of age, an electroencephalogram (EEG) was conducted because of nocturnal episodes of generalized stiffness, demonstrating interictal epilepticform abnormalities. The ictal EEG revealed generalized tonic seizures. Adrenocorticotropic hormone was able to reverse the epileptic encephalopathy. However, two months later, the seizures appeared again and the child is actually severely refractory now. Seizures were difficult to control with topiramate, lamotrigine, or levetiracetam. Magnetic resonance imaging findings were normal. An ophthalmological examination showed a flecked retina; electroretinogram and visual evoked potentials were normal. The first cognitive evaluation detected a borderline cognitive level. However, when the child was eight years old, her EEG and clinical pattern suggested epileptic 
Table 1 Expression levels of selected sub-telomere genes of the ring17 patient, significantly deregulated compared with controls

\begin{tabular}{|c|c|c|c|}
\hline Gene symbol & Gene name & $\begin{array}{c}\text { Fold change } \\
\text { (standard deviation) }\end{array}$ & $P$ \\
\hline \multicolumn{4}{|c|}{$17 p: 1$ to $1,500,000$} \\
\hline RPH3AL & Rabphilin 3A-like (without C2 domains) & $-2.99(0.15)$ & 0.001 \\
\hline FAM57A & Family with sequence similarity 57 , member $\mathrm{A}$ & $-1.61(0.04)$ & 0.002 \\
\hline GEMIN4 & Gem (nuclear organelle) associated protein 4 & $-1.70(0.03)$ & 0.001 \\
\hline RNMTL1 & RNA methyltransferase like 1 & $1.13(0.03)$ & 0.018 \\
\hline NXN & Nucleoredoxin & $-1.87(0.05)$ & 0.001 \\
\hline YWHAE & Tyrosine 3-monooxygenase/tryptophan 5-monooxygenase activation protein, epsilon polypeptide & $-1.04(0.01)$ & $<0.001$ \\
\hline MYO1C & Myosin IC & $-1.15(0.01)$ & 0.001 \\
\hline \multicolumn{4}{|c|}{$17 q: 79,695,210$ to- $81,195,210$} \\
\hline ARHGDIA & $\rho$ GDP dissociation inhibitor a & $-1.67(0.02)$ & $<0.001$ \\
\hline SIRT7 & Sirtuin (silent mating type information regulation 2 homolog) 7 (S. cerevisiae) & $-1.09(0.01)$ & 0.010 \\
\hline NARF & Nuclear prelamin A recognition factor & $-1.86(0.02)$ & $<0.001$ \\
\hline FOXK2 & Forkhead box K2 & $-1.44(0.07)$ & 0.008 \\
\hline FN3K & Fructosamine 3 kinase & $-2.18(0.06)$ & $<0.001$ \\
\hline
\end{tabular}

Genes are listed from the telomere down to $1.5 \mathrm{Mb}$ on the $\mathrm{p}$ arm and from the position $79,695,210$ of the $q$ arm up to the telomere. The expression values are reported as the mean fold change of three replicates.

encephalopathy, and the child experienced cognitive deterioration.

The mother's child had multiple café-au-lait spots on her trunk without facial anomalies or cognitive deficit.

\section{Classical and molecular cytogenetic investigations}

A study of the lymphocytes revealed a mosaic karyotype: 46,XX,r(17)(p13q25)[36]/46,XX[14] (Figure 1A). G-banding analysis of the fibroblasts showed two cell lines, the most abundant clone having a normal karyotype (98\%) and the other having a double ring 17 (2\%). Chromosome analysis of the patient's parents displayed a normal paternal karyotype, and a mosaic ring 17 maternal karyotype: 46,XX,r(17)(p13q25)[22]/46,XX[28]. All the FISH pan-telomeric (Figures $1 \mathrm{~B}, \mathrm{C}$ ) and subtelomeric probes showed clear signals in the patient and her mother, both on the normal chromosome 17 and on the ring. This confirmed that no terminal deletion had occurred. An array-CGH study of both cases did not detect any submicroscopic deletions or duplications. Segregation analysis of the family group revealed that the ring of the patient and her mother derived from the chromosome 17 of the patient's maternal grandmother (Figure 1D). The same study also excluded uniparental disomy because all of the informative markers were found on both the normal and the rearranged chromosomes. Additional microsatellite investigation, performed on fibroblasts of normal skin and a café au lait skin spot biopsy of the patient, displayed the presence of a biallelic pattern of transmission related to each parent (Table 2).

\section{Quantitative fluorescence in-situ hybridization (Q-FISH) analysis}

Chromosome-specific telomere length was assessed in either normal (Figure 2A) or ring metaphases (Figure 2B) obtained from the patient. Data showed that the means of all telomere lengths in normal and ring metaphases (61 and $53 \mathrm{~T} / \mathrm{C} \%$ respectively) did not differ significantly. Moreover, the telomere length patterns in the two metaphase classes were very similar, and in both the shortest telomeres were located at 20q, 16p, 21q, 22q, $19 p, 17 p$, and $17 q$ whereas the longest were located at $\mathrm{Xp}, 4 \mathrm{p}, 21 \mathrm{p}, 12 \mathrm{q}, 8 \mathrm{p}, 14 \mathrm{p}, 1 \mathrm{p}$, and 6q.

Telomere length analysis of chromosome 17 in the patient showed that telomere length in the ring chromosome $(48 \mathrm{~T} / \mathrm{C} \%)$ was significantly shorter than the sum of the telomeres at the p-ter and the q-ter in normal chromosome 17, if scored in normal (104 T/C\%) or ring metaphases (95 T/C\%) (Figure 2C). Conversely, the telomeric DNA in the ring chromosome of the patient's mother $(76 \mathrm{~T} / \mathrm{C} \%)$ was comparable in length to that of the normal chromosome 17 in normal metaphases (77 $\mathrm{T} / \mathrm{C} \%$ ) and in ring metaphases $(67 \mathrm{~T} / \mathrm{C} \%$ ) (Figure $2 \mathrm{C}$ ). As expected, telomeres in the normal chromosome 17 were longer in the patient than in her mother, while telomeres in the ring chromosome were significantly shorter in the patient than in her mother $(48 \mathrm{~T} / \mathrm{C} \%$ and $76 \mathrm{~T} / \mathrm{C} \%$ respectively) (Figure $2 \mathrm{C}$ ).

To compare the ring 17 of the family to those of normal healthy individuals, lymphocytes from nine (four children and five adults) normal donors were also analyzed for telomere length (Table 3). Considering the 


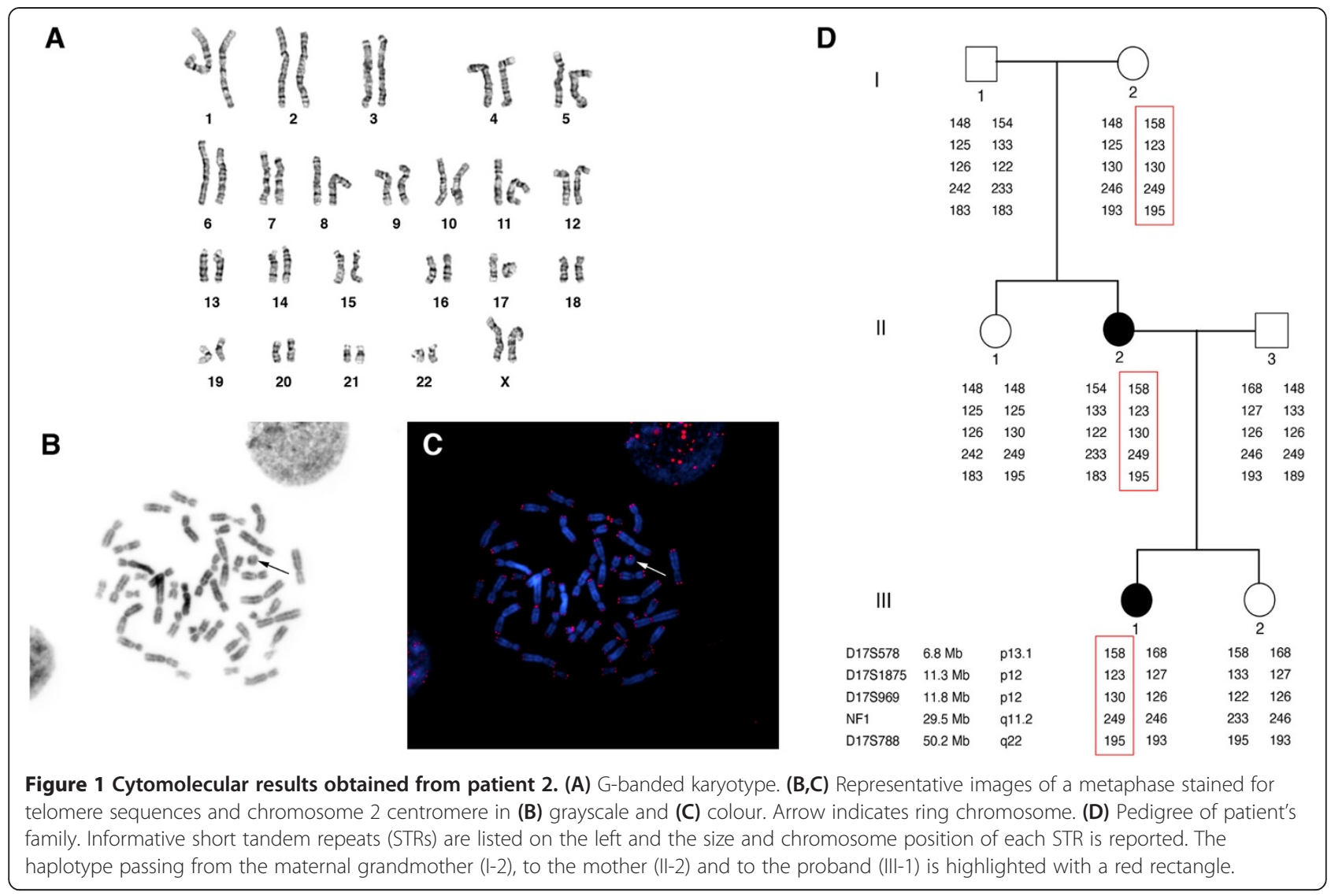

mean cellular telomere length, we found an age-related telomere erosion in the control individuals, with the mean telomere lengths ranging from $94 \mathrm{~T} / \mathrm{C} \%$ in children to $58 \mathrm{~T} / \mathrm{C} \%$ in adults (Figure $2 \mathrm{~F}, \mathrm{G}$ ). The values for the patient's mother and aunt (54 and $50 \mathrm{~T} / \mathrm{C} \%$ respectively) were comparable to the age-matched controls $(58 \mathrm{~T} / \mathrm{C} \%)$, indicating a normal telomere length in both family members. Interestingly, the patient showed reduced telomeric lengths with telomeres much shorter (53 $\mathrm{T} / \mathrm{C} \%$ ) than the other age-matched children $(94 \mathrm{~T} / \mathrm{C} \%)$ (Figure 2F,G).

When we turned our attention to chromosome 17 telomere lengths (ring and normal), we observed the same trend in mean cellular telomere length. Thus, the

Table 2 Segregation analysis of the fibroblasts of the patient

\begin{tabular}{lllll}
\hline & D17S1839 & D17S1301 & D17S752 & D17S788 \\
\hline $\begin{array}{l}\text { III-1 normal and café au } \\
\text { lait skin spot biopsy }\end{array}$ & $234 / 242$ & $148 / 153$ & $162 / 171$ & $195 / 193$ \\
$\begin{array}{l}\text { II-2 maternal peripheral } \\
\text { blood }\end{array}$ & $242 / 245$ & 148 & 162 & $183 / 195$ \\
$\begin{array}{l}\text { II-3 paternal peripheral } \\
\text { blood }\end{array}$ & $234 / 252$ & $153 / 157$ & $158 / 171$ & $189 / 193$ \\
\hline
\end{tabular}

These data clearly show that the proband III-1 inherited one chromosome 17 from her mother (II-2) and the other one from her father (II-3). patient displays telomeres on chromosome 17 (both ring and normal) shorter than those of normal healthy children, whereas the other members of the family were not significantly different in telomere length, as compared with age-matched controls (Figure 2D,E).

\section{Discussion}

Ring chromosomes are rare cytogenetic abnormalities that usually occur as a consequence of breaks and deletions at both ends of the chromosome, with subsequent fusion of the remaining portions. The breakpoints may be located within euchromatic regions, with partial monosomy of the distal ends of the short and long arms of the chromosome, but sometimes they may involve only one or both the telomere repeats. Though uncommon, the last mechanism has been described in the literature as a telomere-telomere fusion with no detectable loss of euchromatin $[14,15]$.

In a previous report [11], we have described the case of a ring 17 chromosome in a young boy, providing an accurate characterization of the defect, which resulted in complete loss of the telomeric ends with the conservation of the whole euchromatic regions. The clinical picture was reported as a mild ring 17 syndrome with moderate intellectual disability, seizures, dysmorphic traits, and café au lait and hypochromic skin spots. We 


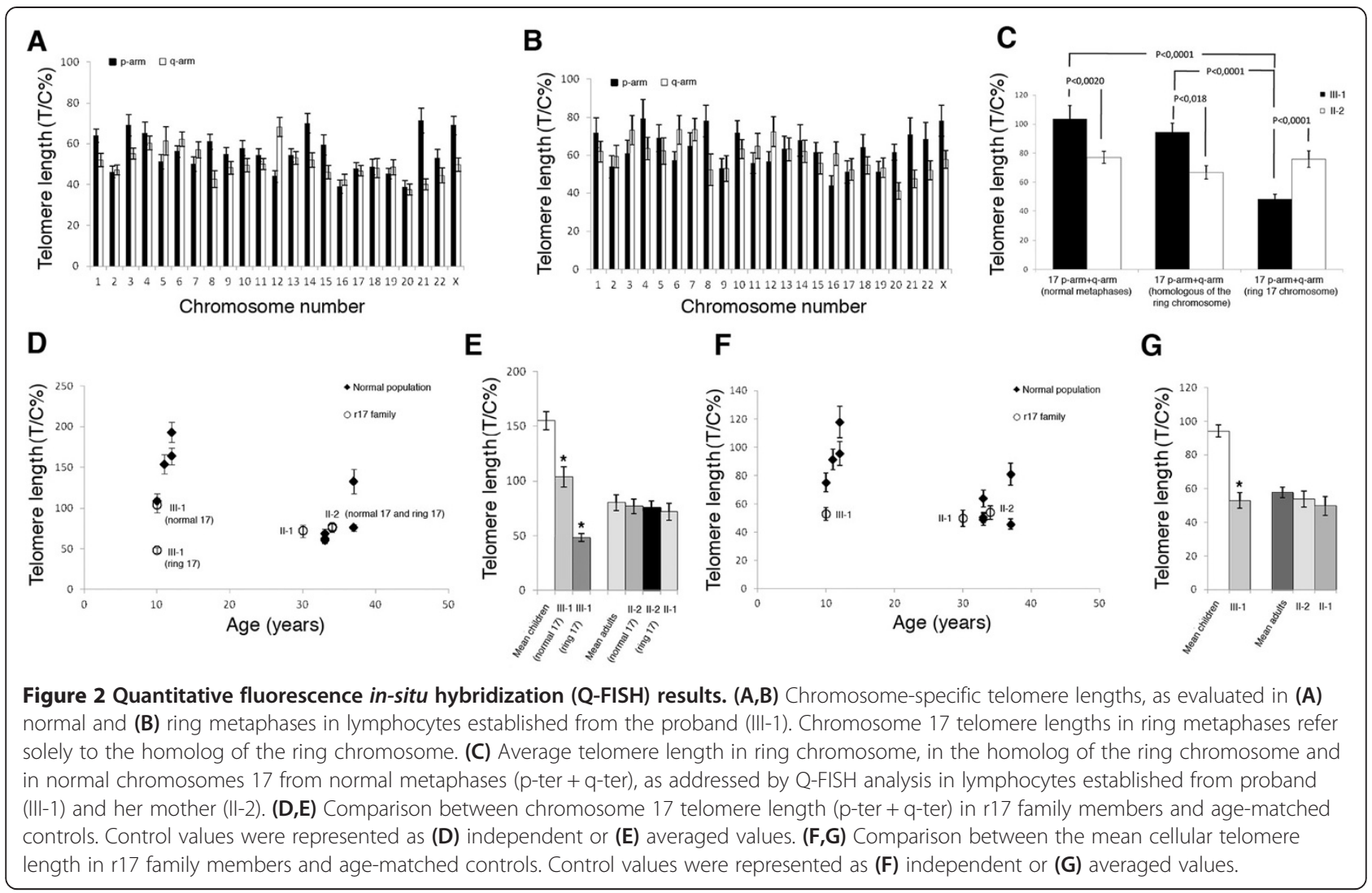

compared this to the most severe lissencephaly syndrome, which involves functional loss of the LIS1 gene.

Several hypotheses have been proposed to explain the relevant phenotype of the mild ring chromosome 17 syndrome, such as 'dynamic mosaicism' or the 'ring syndrome'. However, they have never been supported by clear experimental evidence. It has been suggested that the epigenetic silencing of gene expression through a telomere position effect is one of the alternative mechanisms that occur in ring chromosome syndrome [9]. This silencing effect contributes to an alteration of the function of genes located near the ring fusion point by modulating (mainly downregulating) their expression in a telomere-dependent fashion [10,16-18].

Here, we note that, in the absence of detectable telomere repeats in the first patient [11], a group of noncontiguous genes that were within an interval of $1.5 \mathrm{Mb}$ from the telomeres of $\mathrm{p}$ and $\mathrm{q}$ arms were significantly downregulated compared with the controls. Although the biological functions of these genes are manifold, most of the latter play a key role in cellular and tissue functions (that is, $R P H 3 A L, F N 3 K$, and $N A R F$ ), cell cycle (that is, ARHGDIA), DNA processing and regulation (that is, FOXK2 and GEMIN4) and cell growth and early development (that is, $N X N$ and FAM57A). Our data suggest that the concomitant downregulation of these genes may lead to an imbalance of cell activities and hamper proper physiological interactions, ultimately producing the phenotypic aspects related to ring 17 syndrome. Moreover, the upmodulation of NF1 and the downregulation of LIS1, located outside the studied $1.5 \mathrm{Mb}$ region, reveal that the observed effects are not linearly correlated to the distance from the telomeres.

The second case we studied regards a young girl with a cytogenetic finding of mosaic ring 17 chromosome. The clinical phenotype presents, as in the previous case, the classical features of mild ring 17 syndrome, with the key symptoms being seizures, intellectual disability, and café au lait skin spots. Nevertheless, chromosome and molecular cytogenetic investigations demonstrated that the telomere ends were preserved. Familial studies revealed that the mother was the carrier of a mosaic ring 17 chromosome with the sole phenotypic feature of café au lait skin spots. Ring chromosomes usually follow the conventional method of transmission resulting, when the rearrangement is passed on, in a constitutional ring syndrome with all, or the majority, of cells containing the abnormal chromosome. The familial transmission of a mosaic ring is extremely rare and represents a challenge still to be clarified [1]. The proband's maternal grandparents, sister, and aunt exhibited a normal karyotype in accordance with the data of the segregation 
Table 3 Telomere lengths in members of the ring 17 family and in normal age-matched individuals

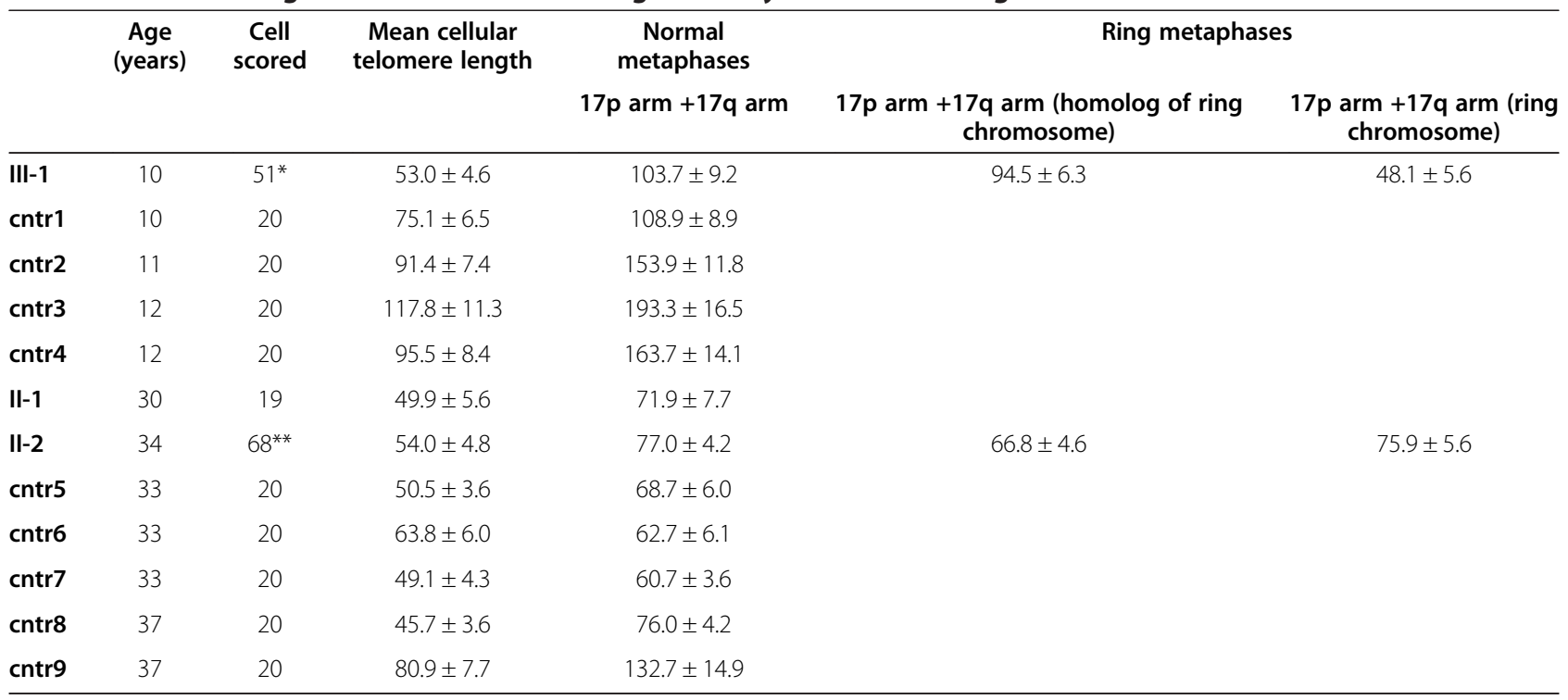

Data are shown for all the individuals analyzed. ${ }^{*} 18$ normal metaphases and 33 carrying the ring 17 chromosome. ${ }^{* *} 46$ normal metaphases and 22 carrying the ring 17 chromosome.

analysis that clearly showed that the ring chromosome derived from the maternal grandmother and was transmitted only to the affected subjects. We also excluded uniparental disomy, since all of the informative markers were found on both the normal and the rearranged chromosomes. Moreover, we tested a set of microsatellites on normal skin and a café au lait skin spot biopsy of the patient, and this showed the presence of a biallelic pattern of transmission related to each parent.

Analysis of telomere lengths in the patient's lymphocytes revealed that telomeres of chromosome 17 were the shortest compared with the other chromosomes of the cell in both normal and ring metaphases (Figure 2A,B). This evidence raises the hypothesis that chromosome 17 could be prone to reaching critical telomere lengths, leading to an increased risk of ring formation.

When we compared the telomere lengths of the ring chromosome of the patient and her mother with the mean telomere lengths of chromosome 17 in age-matched controls, we found that telomeres of the ring chromosome of the patient were at least three times shorter $(48 \mathrm{~T} / \mathrm{C} \%$ vs. $155 \mathrm{~T} / \mathrm{C} \%$ ) (Figure 2D,E), whereas no remarkable differences were found for the patient's mother $(76 \mathrm{~T} / \mathrm{C} \%$ vs. 80 $\mathrm{T} / \mathrm{C} \%$ ). Therefore, assuming that the ring chromosome is generated during prenatal life and that ring chromosome telomeres do not shorten upon cell divisions (as telomeres usually do), we can hypothesize that the generation of the ring chromosome is the result of an extensive loss of telomere repeats at an early stage of development.

The mosaicism rate is another important aspect to consider in determining the phenotypic features of the syndrome. Values less than $10 \%$ do not usually present clinical manifestation, whereas higher rates are associated strictly with different degrees of disease expression. Conversely, this rate tends to decline through one's lifespan. In fact, the rearranged chromosome is preferentially lost during cell divisions, leading to apoptosis and premature cell death. In our case, the patient and her mother showed respectively $72 \%$ and $44 \%$ of mosaic cells in the peripheral blood. Nevertheless, only the patient presented relevant symptoms. The telomere shortening observed in the patient with respect to the mother appears as an additional factor that might have influenced the generation of the ring chromosome and the occurrence of this peculiar phenotype. These hypotheses are also confirmed by the first case that we investigated, which completely lacked the telomeres, as well as previous reports describing ring chromosome 17 with subtelomeric deletions, but conserving the LIS1 gene.

\section{Conclusions}

In summary, our studies clearly outline that telomere length reduction plays a determining role in at least two important aspects of the ring 17 syndrome.

First, the cases we presented show a strong genotypephenotype correlation regarding clinical symptoms and telomeric shortening. Patient 1, with the most severe phenotype, lost the telomeric ends completely. Patient 2, with an intermediate picture, displayed a high shortening of the telomeric repeats, while her mother, affected by a lower reduction of telomere length, presented with only skin spots without any other symptom.

Second, telomere shortening acts as a novel recognized mechanism responsible for ring formation and 
mosaicism assessment. In fact, we demonstrated that the ring chromosome was not selectively lost during the cell cycle, being the autologous maternal linear chromosome present on all cells in the skin biopsy. Moreover, the ring chromosome of the patient's mother, being unable to change its structural characteristic during gametogenesis, has telomeres longer than that of the patient. These observations led us to hypothesize that the chromosome transmitted is the normal autologous one and that, as a consequence of the telomere shortening during embryogenesis, it underwent a circularization process, ultimately leading to the formation of a ring chromosome. The stage in which this occurrence takes place is responsible for the different mosaicism rate.

The influence of telomere shortening and the telomere position effect should also be investigated in other patients carrying the ring chromosome 17; however, since the ring chromosome 17 syndrome is very rare, a big effort by all the scientific community will be necessary to recruit a larger number of patients.

\section{Methods}

\section{Gene expression analysis}

All the samples were collected according to the current version of the Declaration of Helsinki and written informed consent for publication of this manuscript and accompanying images was obtained from the patients' parents and relatives. All experiments were ethically approved by the 'Bambino Gesù' Children's Hospital Scientific Board.

Skin fibroblasts of the patient were obtained from a biopsy of a hypochromic cutaneous lesion using standard methods. Skin fibroblasts of two healthy children were also collected and used as calibrator samples (reference). Total RNA was isolated from fibroblasts with TRIzol (Ambion, Life Technologies, Paisley, UK) according to the manufacturer's protocol. For qPCR analysis, $1 \mu \mathrm{g}$ of total RNA was reverse-transcribed to cDNA using a high-capacity cDNA archive kit (Applied Biosystems, Life Technologies, Paisley, UK). The expression levels of 26 selected genes and one housekeeping gene (GAPDH) of the ring17 patient were measured in each sample by real-time qPCR using pre-designed TaqMan gene expression assays on an ABI PRISM 7900HT Sequence Detection System (Applied Biosystems) according to the manufacturer's protocol, and compared with controls.

The investigations involved 15 genes (ACTG1, GCGR, ARHGDIA, NPB, SIRT7, SECTM1, UTS2R, NARF, FOXK2, RAB4OB, FN3KRP, FN3K, TBCD, ZNF750, METRNL) and 9 genes (DOC2B, RPH3AL, FAM57A, GEMIN4, RNMTL1, NXN, TUSC5, YWHAE, MYO1C) located on the subtelomere region of the long and short arms, respectively, of chromosome 17 encompassing a $1.5 \mathrm{Mb}$ region, starting from each telomere. Two other genes (NF1 and LIS1) placed outside the $1.5 \mathrm{Mb}$ region (17q11.2 and 17p13.3, respectively), were also investigated.

For each sample, three replicates were run for each gene in a 96-well plate. Real-time qPCR reactions were carried out following the manufacturer's instructions. Gene expression values were determined as $\Delta \mathrm{Ct}(\mathrm{Ct}$ $(\mathrm{GAPDH})-\mathrm{Ct}$ (gene)) and relative quantities between different samples were determined as $\Delta \Delta \mathrm{Ct}(\Delta \mathrm{Ct}(\mathrm{pa}-$ tient $)-\Delta C t($ calibrator sample $))$ [19]. All data are expressed as mean \pm standard deviation. The statistical comparison between the ring 17 patient and control individuals was performed using Student's $t$ test at a level of significance of $0.05(P<0.05)$.

\section{Classical and molecular cytogenetic investigations}

Karyotyping with G-banding was performed using standard methods on lymphocytes obtained from peripheral blood and fibroblasts from a biopsy of skin with or without hypochromic cutaneous lesions. Chromosomes were also examined on lymphocytes of both of the patient's parents, as well as her sister, her maternal grandparents and her aunt. The karyotypes were described according to the International System for Human Cytogenetic Nomenclature (ISCN 2009).

To search for possible deletions at telomeric regions, FISH was performed with human pan-telomeric probes, P1 artificial chromosome (CTD-2348K1, 17p13.3) and fosmid (WI-837H17, 17q25.3) clones. The probes were directly labeled with Cy3-dUTP and fluorescein-dUTP (Perkin Elmer Life Sciences, Boston, MA, USA).

Array-CGH was performed using Agilent Technologies Array-CGH Kits (Santa Clara, CA, USA), as described previously [20]. The platform is a 60-mer oligonucleotide-based microarray with an overall median probe spatial resolution of $13 \mathrm{~kb}$. To evaluate whether the copy number variations, detected by array-CGH, were potentially correlated with the clinical phenotype of our patient, bioinformatic analysis was carried out, consulting the Database of Genomic Variants BioXRT (http://projects.tcag.ca/variation/).

\section{Segregation analysis}

DNA was extracted from peripheral blood of the patient and her relatives with a high pure PCR template preparation kit (Roche, Mannheim, Germany), according to the producer's instructions. To assess the familiar origin of the ring 17, a panel of STRs was assembled using multiple primer pairs obtained from the UniSTS database (http:// www.ncbi.nlm.nih.gov/unists/). The STRs analyzed, listed starting from 17pter to 17qter, were: D17S578, D17S1875, D17S969, NF1, D17S788, D17S1306, D17S1819, D17S789, D17S1839, D17S1292. The first five STRs were the informative ones. DNA was amplified by means of a GeneAmp PCR System 2700 (Applied Biosystems, Foster City, CA, 
USA) following the standard protocol. One primer from each pair was fluorescently labeled and PCR products were run on an ABI Prism 310 (Applied Biosystems), using GeneMapper v 3.0 software.

Additional studies were performed also on café au lait skin spots and a normal skin biopsy of the patient to investigate the parental transmission of the chromosome 17.

\section{Q-FISH analysis}

Q-FISH staining was performed as previously described [21] with minor modifications. Briefly, 48 hours after the seeding, slides were rinsed with PBS at $\mathrm{pH} 7.5$, and fixed in $4 \%$ formaldehyde for $2 \mathrm{~min}$. After two rinses in PBS, the slides were incubated in acidified pepsin solution for 10 min, rinsed, and dehydrated through graded alcohols. Slides and probes (Cy3 linked telomeric and chromosome 2 centromeric PNA probe, DAKO Cytomatation, Denmark) were co-denatured at $80^{\circ} \mathrm{C}$ for $3 \mathrm{~min}$ and hybridized for 2 hours at room temperature in a humidified chamber. After hybridization, slides were washed twice for $15 \mathrm{~min}$ in 70\%

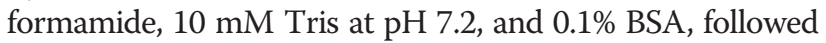
by three 5 -minute washes in $0.1 \mathrm{M}$ Tris at $\mathrm{pH} 7.5,0.15 \mathrm{M}$ $\mathrm{NaCl}$, and $0.08 \%$ Tween 20 . Slides were then dehydrated with an ethanol series and air dried. Finally, slides were counterstained with 4,6-diamidino-2-phenylindole (Sigma Aldrich, St. Louis, MO) in Vectashield (Vector Laboratories, Burlingame, CA). Images were captured at $63 \times$ magnification with an Axio Imager M1 (Carl Zeiss, Germany) equipped with a charge-coupled device camera, and the telomere size was analyzed with ISIS software (MetaSystems, Germany). The software calculates telomere lengths as the ratio between the fluorescence of each telomere signal and the fluorescence of the centromere of chromosome 2, used as the internal reference in each metaphase analyzed. The centromere 2 DNA sequence, which the probe recognizes, has a stable length and can be used as a reference. Data were expressed as a percentage $(\mathrm{T} / \mathrm{C} \%)$ [22]. For each individual, at least 20 metaphases have been analyzed.

\section{Abbreviations \\ BSA: Bovine serum albumin; EEG: Electroencephalogram; FC: Fold change; FISH: Fluorescence in-situ hybridization; PBS: Phosphate-buffered saline; PCR: Polymerase chain reaction; Q-FISH: Quantitative fluorescence in-situ hybridization; qPCR: Quantitative polymerase chain reaction; STR: Short tandem repeat.}

\section{Competing interests}

The authors declare that they have no competing interests.

\section{Authors' contributions}

CS, MCR, PS, LC, and SR performed biological sample processing and cytomolecular investigations. FB and AS carried out Q-FISH analysis. AM and LDS performed gene expression studies. GD, SG, SP, and EP carried out segregation analysis and interpreted the data. MCD, RC, and MEH recruited patients, collected biological samples, and performed clinical evaluations. CS, $F B, A M, M C R, L D S, A S$, and $A A$ analyzed the data and drafted the manuscript. FRL critically reviewed the manuscript. CS, MCR, and AA conceived and designed the study. All authors read and approved the final manuscript.

\section{Author details}

'Cytogenetics and Molecular Genetics Unit, 'Bambino Gesù' Children's Hospital, IRCCS, Piazza S. Onofrio 4, 00165 Rome, Italy. ${ }^{2}$ Department of Biology, University 'Roma Tre', Rome, Italy. ${ }^{3}$ Gene Expression-Microarrays Laboratory, 'Bambino Gesù' Children's Hospital, IRCCS, Rome, Italy. ${ }^{4}$ Medical Genetics Unit, 'Bambino Gesù' Children's Hospital, IRCCS, Rome, Italy. ${ }^{5}$ Neurology Unit, 'Bambino Gesù' Children's Hospital, IRCCS, Rome, Italy. ${ }^{6}$ Dermatology Unit, 'Bambino Gesù' Children's Hospital, IRCCS, Rome, Italy.

Received: 23 September 2013 Accepted: 13 December 2013 Published: 7 January 2014

\section{References}

1. Kosztolanyi G, Méhes K, Hook EB: Inherited ring chromosomes: an analysis of published cases. Hum Genet 1991, 87:320-324.

2. Kosztolanyi G: Does ring syndrome exist? An analysis of 207 case reports on patients with a ring autosome. Hum Genet 1987, 75:175-179.

3. Tham WH, Zakian VA: Transcriptional silencing at Saccharomyces telomeres: implications for other organisms. Oncogene 2002, 21:512-521.

4. Fisher J, Upadhyaya M: Molecular genetics of facioscapulohumeral muscular dystrophy (FSHD). Neuromuscul Disord 1997, 7:55-62.

5. Baur JA, Zou Y, Shay JW, Wright WE: Telomere position effect in human cells. Science 2001, 292:2075-2077.

6. van Geel M, Dickson MC, Beck AF, Bolland DJ, Frants RR, van der Maarel SM, de Jong PJ, Hewitt JE: Genomic analysis of human chromosome 10q and 4q telomeres suggests a common origin. Genomics 2002, 79:210-217.

7. Inoue K, Osaka H, Thurston VC, Clarke JT, Yoneyama A, Rosenbarker L, Bird TD, Hodes ME, Shaffer LG, Lupski JR: Genomic rearrangements resulting in PLP1 deletion occur by nonhomologous end joining and cause different dysmyelinating phenotypes in males and females. Am J Hum Genet 2002, 71:838-853.

8. Clapp J, Bolland JD, Hewitt JE: Genomic analysis of facioscapulohumeral muscular dystrophy. Brief Funct Genomic Proteomic 2003, 2:213-223.

9. van Karnebeek CD, Quik S, Sluijter S, Hulsbeek MM, Hoovers JM, Hennekam RC: Further delineation of the chromosome 14q terminal deletion syndrome. Am J Med Genet 2002, 110:65-72.

10. Jalal SM, Harwood AR, Sekhon GS, Pham Lorentz C, Ketterling RP, BabovicVuksanovic D, Meyer RG, Ensenauer R, Anderson MH Jr, Michels W: Utility of subtelomeric fluorescent DNA probes for detection of chromosome anomalies in 425 patients. Genet Med 2003, 5:28-34.

11. Surace C, Piazzolla S, Sirleto P, Digilio MC, Roberti MC, Lombardo A, D'Elia G, Tomaiuolo AC, Petrocchi S, Capolino R, El Hachem M, Claps Sepulveda D, Sgura A, Angioni A: Mild ring 17 syndrome shares common phenotypic features irrespective of the chromosomal breakpoints location. Clin Genet 2009, 76:256-262.

12. Havlovicova M, Novotna D, Kocarek E, Novotna K, Bendova S, Petrak B, Hrdlicka M, Sedlacek Z: A girl with neurofibromatosis type 1, atypical autism and mosaic ring chromosome 17. Am J Med Genet Part A 2007, 143:76-81.

13. Vazna A, Havlovicova M, Sedlacek Z: Molecular cloning and analysis of breakpoints on ring chromosome 17 in a patient with autism. Gene 2008, 407:186-192.

14. Vermeesch JR, Baten E, Fryns JP, Devriendt K: Ring syndrome caused by ring chromosome 7 without loss of subtelomeric sequences. Clin Genet 2002, 62:415-417.

15. Le Caignec C, Boceno M, Jacquemont S, Nguyen The Tich S, Rival JM, David A: Inherited ring chromosome 8 without loss of subtelomeric sequences. Ann Genet 2004, 47:289-296.

16. Perrod S, Gasser SM: Long range silencing ad position effects at telomeres and centromeres: parallels and differences. Cell Mol Life Sci 2003, 60:2303-2318.

17. Bickmore WA, van der Maarel SM: Perturbations of chromatin structure in human genetic disease: recent advances. Hum Mol Genet 2003, 12:R207-R213

18. Pedram M, Sprung CN, Gao Q, Lo AW, Reynolds GE, Murnane JP: Telomere position effect and silencing of transgenes near telomeres in the mouse. Mol Cell Biol 2006, 26:1865-1878.

19. Livak KJ, Schmittgen TD: Analysis of relative gene expression data using real-time quantitative $P C R$ and the $2(-\Delta \Delta C(T))$ method. Methods 2001, 25:402-408. 
20. Roberti MC, Surace C, Digilio MC, D'Elia G, Sirleto P, Capolino R, Lombardo A, Tomaiuolo AC, Petrocchi S, Angioni A: Complex chromosome rearrangements related $15 q 14$ microdeletion plays a relevant role in phenotype expression and delineates a novel recurrent syndrome. Orphanet J Rare Dis 2011, 19:1750-1172.

21. Berardinelli F, Antoccia A, Cherubini R, De Nadal V, Gerardi S, Cirrone GA, Tanzarella C, Sgura A: Transient activation of the ALT pathway in human primary fibroblasts exposed to high-LET radiation. Radiat Res 2010, 174:539-549,

22. Perner S, Brüderlein S, Hasel C, Waibel I, Holdenried A, Ciloglu N, Chopurian H, Nielsen KV, Plesch A, Högel J, Möller P: Quantifying telomere lengths of human individual chromosome arms by centromere-calibrated fluorescence in situ hybridization and digital imaging. Am J Pathol 2003, 163:1751-1756.

doi:10.1186/1756-8935-7-1

Cite this article as: Surace et al:: Telomere shortening and telomere position effect in mild ring 17 syndrome. Epigenetics \& Chromatin 2014 7:1.

\section{Submit your next manuscript to BioMed Central} and take full advantage of:

- Convenient online submission

- Thorough peer review

- No space constraints or color figure charges

- Immediate publication on acceptance

- Inclusion in PubMed, CAS, Scopus and Google Scholar

- Research which is freely available for redistribution 